УДК 669-1

\title{
Absorption of Ultrasound in Manganese Steel 20HL
}

\author{
Anastasiya A. Karavaitseva ${ }^{a}$, Ludmila I. Kveglis ${ }^{a}$, \\ Sergei S. Gert ${ }^{\mathrm{b}}$ and Vladislav V. Anfilofiev ${ }^{\mathrm{b}}$ \\ ${ }^{a}$ Siberian Federal University \\ 79 Svobodny, Krasnoyarsk, 660041, Russia \\ ${ }^{b}$ Sarsen Amanzholov East-Kazakhstan State University \\ 34, 30th Guards Division Str., Ust-Kamenogorsk, \\ 070002, Republic of Kazakhstan
}

Received 20.03.2018, received in revised form 12.10.2018, accepted 27.02.2019

In this work, we discovered anomalies in the behavior of acoustic waves in steel 20GL. Correlation between speed of ultrasound and the size of granule set at different temperatures.

Keywords: Damping of ultrasound, plastic deformation, 20GL steel, microstructure, temperature dependence.

Citation: Karavaitseva A.A., Kveglis L.I., Gert S.S., Anfilofiev V.V. Absorption of ultrasound in manganese steel 20HL, J. Sib. Fed. Univ. Eng. technol., 2019, 12(3), 356-365. DOI: 10.17516/1999-494X-0143.

\section{Поглощение ультразвука в марганцовистой стали 20ГЛ}

\author{
А.А. Каравайцева ${ }^{a}$, Л.И. Квеглис ${ }^{\text {, }}$ \\ С.С. Герт ${ }^{\tilde{0}}$ В.В. Анфилофьев ${ }^{\sigma}$ \\ ${ }^{a}$ Сибирский федеральный университет \\ Россия, 660041, Красноярск, пр. Свободнылй,79 \\ ${ }^{6}$ Восточно-Казахстанский государственный \\ университет им. С. Аманжолова \\ Республика Казахстан, 070002, Усть-Каменогорск, \\ ул. 30-й Гвардейской дивизии, 34
}

В данной работе обнаружень аномалии в поведении акустических волн в стали 20 ГЛ. Зависимость между скоростью ультразвука и размером зерна установлена при разньх meмnepamypax.

(C) Siberian Federal University. All rights reserved

This work is licensed under a Creative Commons Attribution-NonCommercial 4.0 International License (CC BY-NC 4.0).

* Corresponding author E-mail address: anastasiya-romanova-16.09@mail.ru 
Ключевые слова: поглощение ультразвука, пластическая деформачия, сталь 20ГЛ, микроструктура, температурная зависимость.

\section{Введение}

Использование ультразвука позволяет производить обработку давлением металлических деталей, используя меньшее давление, следовательно, быстрее и дешевле. То есть можно эффективно использовать ультразвук при интенсивной пластической деформации. Поглощение в однородных твердых телах как ультразвуковых волн, так и упругих волн более низких частот, пропорционально квадрату частоты. Однако твердые тела однородны для ультразвуковых волн только до тех пор, пока неоднородности, имеющиеся в них (а эти неоднородности всегда есть), значительно меньше длины волны. Исследования показывают, что даже такие кажущиеся однородными тела, как железо, имеют в действительности крайне неоднородное строение. [1]. Однородность структуры металлов и сплавов достигается измельчением, вплоть до возникновения наноструктурных состояний, что может быть обеспечено интенсивной пластической деформацией (ИПД) [2]. Интенсивную пластическую деформацию лучше всего проводить в таких условиях, когда достигнут максимальный коэффициент поглощения ультразвуковых волн в веществе. Исследование поглощения ультразвука в металлах в процессе их пластической деформации представляет практический интерес при обработке металлов давлением с целью снижения усилия деформации [3].

\section{Проблема исследования}

Сталь 20ГЛ используется для изготовления ответственных изделий сложного сечения, используемых в железнодорожном транспорте, поэтому необходимо выявить оптимальные условия ее механической обработки: условия, при которых коэффициент поглощения ультразвуковых волн позволит проводить интенсивную пластическую деформацию с минимальной нагрузкой [4]. Такие изделия работают в условиях пониженных температур и знакопеременных нагрузок. При неправильной заливке стали в сечениях образовываются неоднородности кристаллического строения, трещины и раковины, которые выявляются методами неразрушающего контроля. Эти дефекты можно устранить посредством интенсивной пластической деформации.

Цель работы. Исследовать возможность поглощения ультразвука в стали 20ГЛ, выявить причину данного эффекта. Дать теоретическое обоснование наблюдаемым явлениям.

Задачи. 1. Установить причину и дать объяснение поглощения ультразвуковых волн в стали. 2. Найти качественный способ применения эффекта поглощения.

\section{Образцы и методы}

В качестве образцов для исследования использовали исходную, литую сталь 20ГЛ. Для получения серии образцов с нарастающим размером структурных элементов их подвергли термической обработке при температуре $900{ }^{\circ} \mathrm{C}$, задавая разное время выдержки в муфельной печи, и дальнейшему охлаждению на воздухе. Микроструктуру стали исследовали на оптиче- 
ском металлографическом микроскопе сх-4 при увеличении х100 и х500 крат. Скорость ультразвука измеряли методом автоциркуляции импульсов на ультразвуковом толщиномере А1209. Микроструктуру и размер зерна определяли по ГОСТ 5639-82.

Для измерения скорости распространения поверхностной волны в стали поверхность образца делили на прямоугольные участки, соответствующие размеру датчика (расстояние между пьезопреобразователями 33 мм). В каждом участке скорость определяли 5-7 раз, затем находили среднее значение.

\section{Теория}

Специфика структурообразования и деформационного упрочнения при переходе к интенсивным пластическим деформациям впервые была отмечена и систематически изучена в работах В.И. Трефилова, Ю.И. Мильмана и С.А. Фирстова [5-8]. В.И. Трефилов и С.А. Фирстов с сотрудниками обнаружили, что с ростом деформации в материале формируется качественно новый вид дислокационной структуры - сильно разориентированные ячейки. Основная их особенность - это непрерывное увеличение углов разориентировки с ростом деформации при слабом уменьшении поперечных размеров. Как потом оказалось, подобная эволюция структуры присуща и другим металлам. В.И. Трефилов с сотрудниками предложил оценивать деформационное упрочнение при интенсивных деформациях с позиций уменьшения размера зерна - в предельном случае до размера сильно разориентированной ячейки. Его точка зрения о радикальном изменении механизма упрочнения получила многочисленные подтверждения и в настоящее время признается многими учеными. Исходя из сказанного, можно заключить, что направленное и непрерывно происходящее в процессе пластической деформации движение дисклинаций должно приводить к фрагментации структуры металла, т. е. разбиению его на микрообласти, разориентированные на углы порядка нескольких градусов. Чем больше будет степень пластической деформации, тем мельче должны быть фрагменты и больше их разворот относительно друг друга [9].

Уникальной особенностью ультразвуковых методов является то, что при температурах, близких к температуре фазового перехода второго рода в магнитных системах и системах, демонстрирующих структурные фазовые переходы, происходит аномально сильное поглощение ультразвука [4], наглядно выявляемое экспериментально. Оно обусловлено эффектами взаимодействия низкочастотных акустических колебаний с долгоживущими флуктуациями параметра порядка, которые посредством спин-фононного взаимодействия магнитострикционной природы создают случайную силу, приводящую к возмущению нормальных акустических мод. При этом можно выделить релаксационный и флуктуационный механизмы поглощения. Релаксационный механизм, обусловленный динамической линейной связью звуковых волн с параметром порядка, проявляется только в упорядоченной фазе, где статистическое среднее значение параметра порядка отлично от нуля. Поскольку релаксация параметра порядка вблизи точки фазового перехода происходит очень медленно, данный механизм играет существенную роль в диссипации низкочастотных акустических колебаний. Флуктуационный механизм поглощения, определяемый квадратичной связью деформационных переменных в гамильтониане системы с флуктуациями параметра порядка, проявляется во всей критической температурной области. К настоящему времени существует значительное число работ, посвященных 
теоретическому описанию возникающих ультразвуковых аномалий в конденсированных средах при фазовых переходах и дающих адекватное объяснение результатам экспериментальных исследований [10].

Одной из наиболее интересных и важных задач как с экспериментальной, так и теоретической точки зрения является задача исследования влияния дефектов структуры на характеристики распространения ультразвука в материалах, испытывающих фазовые превращения. Структурный беспорядок, обусловленный присутствием примесей или других дефектов структуры, наличие в эффективном гамильтониане нескольких типов конкурирующих взаимодействий, задающих состояние сложной системы, зачастую играют важную роль в поведении реальных материалов и физических систем. Эти факторы могут индуцировать новые типы фазовых переходов, задавать новые классы универсальности критического поведения, модифицировать кинетические свойства систем и обусловливать низкочастотные особенности в динамике системы.

\section{Результаты исследований и их обсуждение}

Для изучения влияния магнитных параметров на механические и ультразвуковые характеристики литой стали 20ГЛ были изготовлены и термически обработаны экспериментальные образцы с размером зерна, начиная с 1, заканчивая 9-м баллом. Образцы были подвергнуты пластической деформации посредством удара маятникового копра при испытании образцов на ударную вязкость.

Металлографические исследования поверхности полученных образцов при увеличении х 500 показаны на рис. 1 , номера 0 - 5 соответствуют номеру образца согласно режиму термообработки.

Затем провели замеры скорости распространения ультразвуковой волны в продольном и поперечном направлениях образцов при комнатной и пониженной температуре (минус $60{ }^{\circ} \mathrm{C}$, согласно ГОСТ 32400-2013), испытания на ударную вязкость $\mathrm{KCV}^{-60}$ (табл. 1).

Как видно из данных табл. 1, ударная вязкость не имеет прямой зависимости от скорости ультразвука или от размера зерна. Однако, построив график зависимости значений скорости ультразвука и балла зерна, можно увидеть некую зависимость указанных параметров в виде зигзагообразной кривой, причем как при комнатной температуре, так и при температуре минус $60{ }^{\circ} \mathrm{C}$ (рис. 2). Наблюдаемая зависимость от размера зерна согласуется с известным уравнением Холла-Петча

$$
\sigma_{\mathrm{T}}=\sigma_{0}+\mathrm{kd}^{-1 / 2}
$$

и уравнением Петча

$$
\beta \mathrm{T}_{\mathrm{xp}}=\ln \beta-\ln C-\operatorname{lnd} \mathrm{d}^{-1 / 2},
$$

где $\sigma_{\mathrm{T}}-$ предел текучести; $\sigma_{0}$ - напряжение трения при движении дислокаций внутри зерен; $\mathrm{d}$ - диаметр зерна, мм; $\mathrm{k}$ - коэффициент пропорциональности; $\mathrm{T}_{\mathrm{xp}}-$ температура хрупкого перехода; $\beta$ и $\mathrm{C}$ - постоянные.

Измерение скорости ультразвука показало, что разброс ее значений в стали составляет 0,2-0,7 \% в зависимости от температуры обработки. Неодинаковые значения скорости ультра- 
0

1

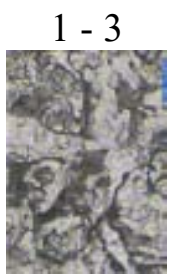

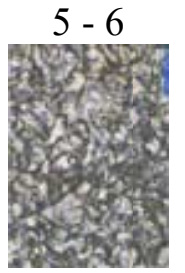

2 Балл зерна

3 $6-7$

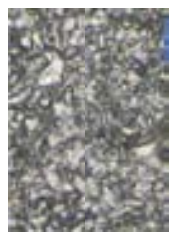
7

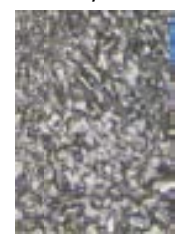

4

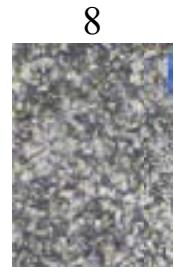

5

9

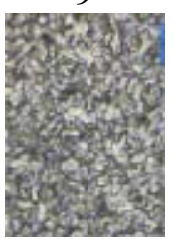

Рис. 1. Эволюция структуры экспериментальных образцов под воздействием температуры в зависимости от времени выдержки

Fig. 1. The evolution of the structure of experimental samples under the influence of temperature depending on the exposure time

Таблица 1. Динамика изменения скорости ультразвука от размера зерна и измерение ударной вязкости

Table 1. Dynamics of ultrasound velocity change from grain size and measurement of toughness

\begin{tabular}{|c|c|c|c|c|c|}
\hline № образца & $\mathrm{t},{ }^{\circ} \mathrm{C}$ & $\begin{array}{c}9 \text { ультразвука, } \\
\text { продольная } \\
\text { (Частота } 4 \text { МГц) }\end{array}$ & $\begin{array}{c}\vartheta \text { ультразвука, } \\
\text { поперечная } \\
\text { (Частота } 10 \text { МГц) }\end{array}$ & 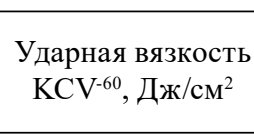 & $\begin{array}{c}\text { Балл зерна, } \\
\text { G }\end{array}$ \\
\hline \multirow{2}{*}{0} & +20 & 5955 & 5975 & \multirow{2}{*}{8,9} & \multirow{2}{*}{$1-3$} \\
\hline & -60 & 6017 & 6079 & & \\
\hline \multirow{2}{*}{1} & +20 & 5978 & 5975 & \multirow{2}{*}{40,1} & \multirow{2}{*}{$5-6$} \\
\hline & -60 & 6039 & 6075 & & \\
\hline \multirow{2}{*}{2} & +20 & 5987 & 5967 & \multirow{2}{*}{45,86} & \multirow{2}{*}{$6-7$} \\
\hline & -60 & 6062 & 6081 & & \\
\hline \multirow{2}{*}{3} & +20 & 5857 & 5957 & \multirow{2}{*}{37,08} & \multirow{2}{*}{7} \\
\hline & -60 & 5936 & 6127 & & \\
\hline \multirow{2}{*}{4} & +20 & 5927 & 5962 & \multirow{2}{*}{39,42} & \multirow{2}{*}{8} \\
\hline & -60 & 5991 & 6075 & & \\
\hline \multirow{2}{*}{5} & +20 & 5930 & 5965 & \multirow{2}{*}{47,51} & \multirow{2}{*}{9} \\
\hline & -60 & 6012 & 6087 & & \\
\hline
\end{tabular}

звука наблюдаются в продольном и поперечном сечениях образца: $V_{R}{ }^{\text {пр }}>V_{R}{ }^{\text {пा }}$, что отражает анизотропию структуры и свойств деформированного металла.

Течение пластической деформации стали 20ГЛ в процессе испытания на растяжение идет неравномерно, это отражается на графике разрушения образца, данный процесс описывается в работе [11].

График зависимости скорости ультразвука от балла зерна при температуре минус $60{ }^{\circ} \mathrm{C}$ имеет более резкий спад и подъем; это связано с тем, что при уменьшении температуры скорость распространения ультразвука увеличивается [12]. Проанализировав полученную зависимость, можно сделать вывод, что при размере зерна 6-7 баллов и частоте 4 МГц скорость ультразвука заметно снижается. Вероятно, в данном месте происходит эффект поглощения ультразвука. Это связано с процессом кристаллизации, так как при 6-7 баллах зерна в микроструктуре стали 20ГЛ наблюдаются крупные ферритные зерна, скопления ферритных зерен и 


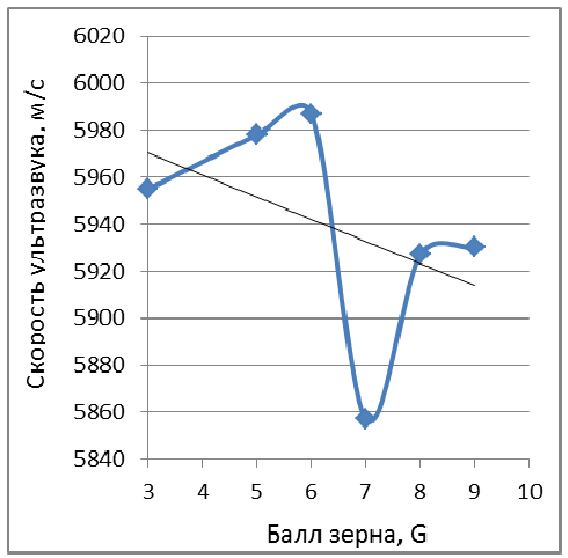

a) при $20{ }^{\circ} \mathrm{C}$

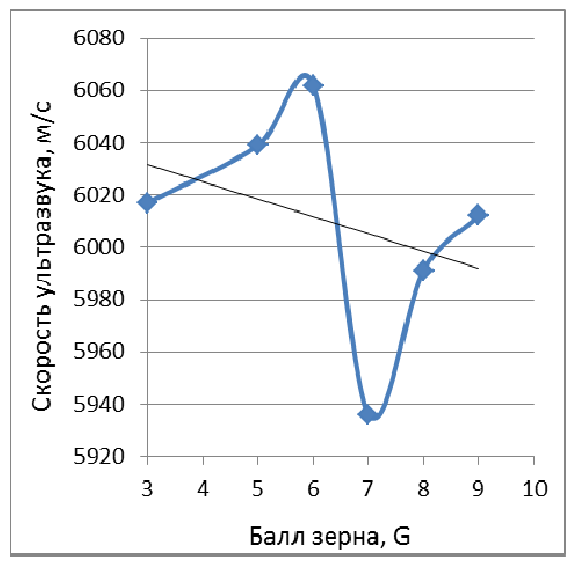

б) при минус $60{ }^{\circ} \mathrm{C}$

Рис. 2. Обнаруженная зависимость скорости ультразвука от балла зерна

Fig. 2. The detected dependence of the speed of ultrasound on the grain score

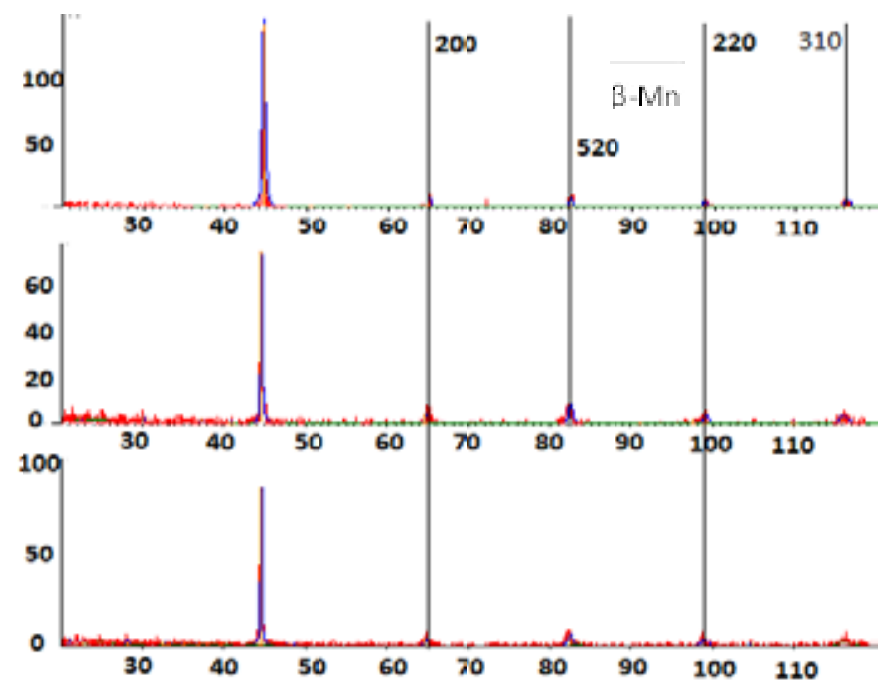

Рис. 3. Рентгенограммы образца № 3. Сверху-вниз: до обработки током, после обработки током, под углом $90^{\circ}$ по отношению к направлению тока

Fig. 3. Radiographs of sample number 3. Top-down: before current treatment, after current treatment, at an angle of $90^{\circ}$ with respect to the direction of current

ферритная сетка. То есть наблюдается структурная неоднородность с одновременным уменьшением ультразвуковой проницаемости.

С целью релаксации внутренних напряжений, в результате которой может уменьшаться поглощение ультразвука, наблюдаемое в образце № 3 (рис. 2) стали 20ГЛ, провели обработку образцов воздействием импульсного тока плотностью $100 \mathrm{~A} / \mathrm{Mм}^{2}$. В работе [13] импульсный ток при определенных режимах может быть использован для ускоренной релаксации внутренних напряжений. Затем снова провели испытания ультразвуком, в результате которого выявлено еще более сильное поглощение ультразвука (три новые точки на рис. 2). Исследования с 
Таблица 2. Межплоскостные расстояния $\alpha-\mathrm{Fe}$ и $\beta-\mathrm{Mn}$

Table 2. Interplanar distances $\alpha-\mathrm{Fe}$ and $\beta-\mathrm{Mn}$

\begin{tabular}{|c|c|c|c|c|c|}
\hline \multicolumn{2}{|c|}{$\boldsymbol{\alpha - F e}$} & \multicolumn{3}{c|}{$\boldsymbol{\beta}$-Mn } & Int. \\
\hline $\mathbf{h ~ k ~ l}$ & $\mathbf{d}$ & Int. & h k l & d & 0.07 \\
\hline 110 & 2.01 & 1.00 & 210 & 2.81 & 1.00 \\
\hline 200 & 1.428 & 0.15 & 221 & 2.10 & 0.66 \\
\hline 211 & 1.166 & 0.38 & 310 & 2.00 & 0.27 \\
\hline 220 & 1.010 & 0.10 & 311 & 1.90 & 0.07 \\
\hline 310 & 0.904 & 0.08 & 321 & 1.68 & 0.03 \\
\hline 222 & 0.825 & 0.03 & 411 & 1.485 & 0.03 \\
\hline 321 & 0.764 & 0.10 & 420 & 1.410 & 0.30 \\
\hline 330 & 0.673 & 0.03 & 510 & 1.237 & 0.20 \\
\hline 420 & 0.638 & 0.03 & 520 & 1.170 & 0.03 \\
\hline
\end{tabular}

Таблица 3. Химический состав стали 20ГЛ

Table 3. The chemical composition of steel 20GL

\begin{tabular}{|c|c|c|c|c|c|c|c|c|}
\hline \multicolumn{9}{|c|}{ Массовая доля элементов } \\
\hline \multirow{2}{*}{$\mathrm{C}$} & \multirow{2}{*}{$\mathrm{Si}$} & \multirow{2}{*}{$\mathrm{Mn}$} & $\mathrm{S}$ & $\mathrm{P}$ & $\mathrm{Cr}$ & $\mathrm{Ni}$ & $\mathrm{Cu}$ & \multirow{2}{*}{$\mathrm{Al}$} \\
\hline & & & \multicolumn{5}{|c|}{ Не более } & \\
\hline $0,17-0,25$ & $0,30-0,50$ & $1,10-1,40$ & $\begin{array}{l}0,030^{1)} \\
0,030^{2)} \\
0,020^{3)}\end{array}$ & $\begin{array}{l}0,040^{1)} \\
\left.0,030^{2}\right) \\
\left.0,020^{3}\right)\end{array}$ & 0,30 & 0,30 & 0,60 & $0,02-0,06$ \\
\hline
\end{tabular}

помощью рентгеновской дифракции проводили на образце № 3 после испытаний ультразвуком до обработки током и после.

Сопоставляя рефлексы на рентгенограмме (рис. 3) с данными табл. 2, можно заключить что рефлекс (221) $\beta$-марганца можно сравнить по интенсивности с рефлексом (110) $\alpha$-железа. Оба рефлекса имеют интенсивность $100 \%$, если рассматривать однокомпонентные материалы. Атомные факторы рассеяния этих материалов практически одинаковы, поскольку они расположены рядом в периодической таблице химических элементов.

Взяв интенсивность рефлекса (110) $\alpha$-железа равной $100 \%$, можно считать, что интенсивность рефлекса (221) $\beta$-марганца составит 4,8 \%. Такая оценка позволяет полагать, что в сплаве сталь 20ГЛ Мn содержится более чем в 2 раза больше, чем в исходном составе (табл. 3).

Изучив образец № 3 до и после обработки импульсным током (рис. 3), можно считать, что такая обработка приводит к образованию дополнительных частиц $\mathrm{Mn}$.

На рис. 4 приведены изображения, полученные в сканирующем электронном микроскопе с микроанализатором INCA Energy. На рис. 4а показано изображение, полученное в лучах марганца Ка, на рис. 4 в в лучах углерода, на рис. 4в - в лучах железа. 


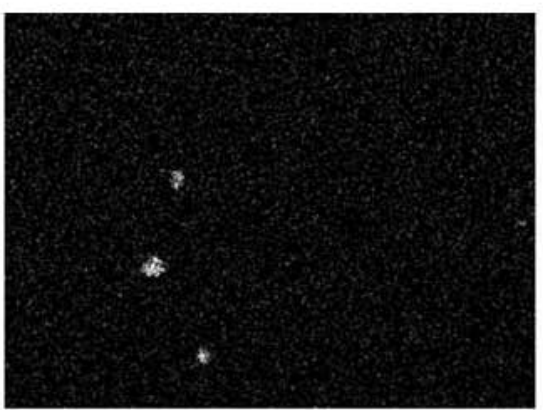

Mn Ka1

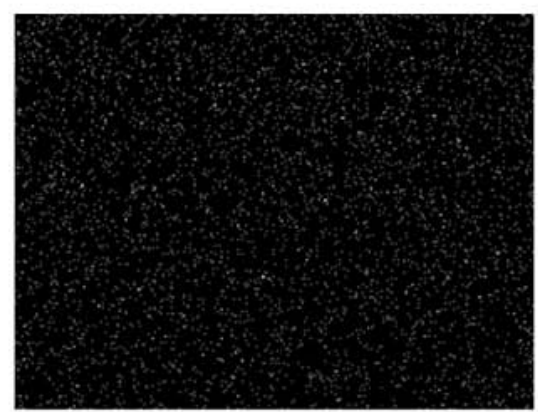

CKa1_2 a)

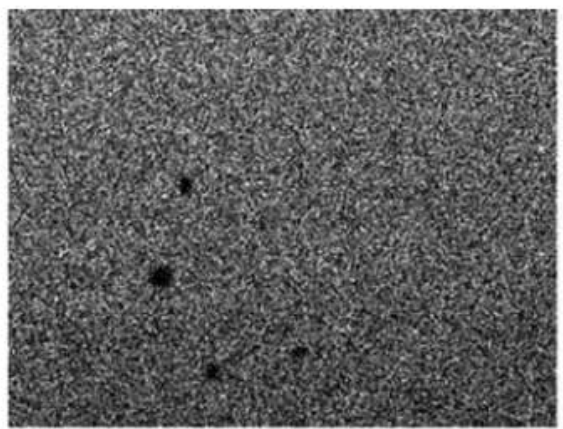

Fe Ka1 б)

в)

Рис. 4. Изображения, полученные в сканирующем электронном микроскопе

Fig. 4. Images obtained in a scanning electron microscope

Микрозондовый анализ (рис. 4) показал, что около 2 \% площади занимают частицы марганца. Положение этих частиц не ассоциируется с положением частиц углерода - на изображении в лучах углерода эти места темные. Следовательно, происхождение частиц марганца не связано с выплавкой стали, поскольку при выплавке марганец легко образует карбиды марганца, значит, появление частиц марганца после прохождения тока высокой плотности может быть связано с реакцией трансмутации железа 57 в марганец 55. Известно, что в любом образце железа существует 2 \% изотопа железа 57. Такая реакция возможна [14].

\section{Выводы}

1. Обнаружены аномалии в поведении акустических волн в сплаве сталь 20ГЛ.

2. Выявлено образование дополнительных частиц марганца после обработки импульсным током стали 20ГЛ.

3. Полученные результаты могут быть использованы для создания технологии обработки изделий сложной формы из стали 20ГЛ.

\section{Список литературы}

[1] Красильников В.А. Звуковые и ультразвуковые волны в воздухе, воде и твердых телаx. М.: Изд-во физико-математической литературы, 1960. 560 с. [Krasilnikov V.A. Sound and

$$
-363-
$$


ultrasonic waves in air, water and solids. M.: Publishing house of physical and mathematical literature, 1960. 560 p. (in Russian)]

[2] Панкратов Д.Л., Шибаков В.Г., Андреев А.П., Андреева С.И., Ситдиков И.М. Исследование разрушения образцов, полученных интенсивной пластической деформацией выдавливанием. Фундаментальные исследования, 2015, 5-3, 531-537 [Pankratov D.L., Shibakov V.G., Andreev A.P., Andreeva S.I., Sitdikov I.M. Investigation of the destruction of samples obtained by severe plastic deformation by extrusion, Fundamental research, 2015, 5-3, 531-537 (in Russian)]

[3] Кулемин А.В. Поглощение ультразвука в металлах в процессе их пластической деформации. Акустический журнал, 1980, 26, 5, 735-740 [Kulemin A.V. Absorption of ultrasound in metals during their plastic deformation, ACOUSTIC JOURNAL, 1980, 26, 5, 735-740 (in Russian)]

[4] Прудников П.В., Прудников В.В., Носихин Е.А. Аномальное поглощение ультразвука в твердых телах при фазовых переходах второго рода с учетом эффектов релаксации. Акустические методы исследования структуры и свойств через частотные и температурные зависимости коэффициента поглощения ультразвука. Физика металлов и металловедение, 2007,104, 3, 235-240 [Prudnikov P.V., Prudnikov V.V., Nosikhin E.A. Anomalous absorption of ultrasound in solids during phase transitions of the second kind, taking into account relaxation effects. Acoustic methods for studying the structure and properties through the frequency and temperature dependences of the absorption coefficient of ultrasound, Metal Physics and Metallography, 2007,104, 3, 235-240 (in Russian)]

[5] Трефилов В.И. Мильман Ю.В., Фирстов С.А. Физические основы прочности тугоплавких металлов. Киев: Наукова думка, 1975. 315 с. [Trefilov V.I. Milman Yu.V., Firstov S.A. Physical bases of strength of refractory metals, Kiev: Naukova Dumka, 1975, 315 p. (in Russian)]

[6] Трефилов В.И., Мильман Ю.В., Иващенко Р.К. Структура, текстура и механические свойства деформированных сплавов молибдена. Киев: Наукова думка, 1983. 232 с. [Trefilov V.I., Milman Yu.V., Ivashchenko R.K. Structure, texture and mechanical properties of deformed molybdenum alloys, Kiev: Naukova Dumka, 1983. 232 p. (in Russian)]

[7] Рубцов А.С., Рыбин В.В. Структурные особенности пластической деформации на стадии локализации течения. Физика металлов и металловедение, 1977, 44, 3, 611-621. [Rubtsov A.S., Rybin V.V. Structural features of plastic deformation at the stage of localization of the flow, Physics of metals and metallography, 1977, 44, 3, 611-621 (in Russian)]

[8] Манилов В.А., Ткаченко В.Г., Трефилов В.И., Фирстов С.А. Структурные изменения в хроме при деформации, Металльь, 1967, 2, 114-122. [Manilov V.A., Tkachenko V.G., Trefilov V.I., Firstov S.A. Structural changes in chromium during deformation, Metals, 1967, 2, 114-122 (in Russian)]

[9] Рыбин В.В. Большие пластические деформациии разрушение металлов. М.: Металлургия, 1986. 226 c. [Rybin V.V. Large plastic deformation and destruction of metals, Moscow: Metallurgy, 1986. 226 p. (in Russian)]

[10] Ландау Л.Д., Халатников И.М. ДАН СССР, 1954. Т. 96.496 с. [Landau L.D., Khalatnikov I.M. DAN USSR, 1954. T. 96, 496 p. (in Russian)]

[11] Романова А.А., Павлов А.В., Немцев И.В. Квеглис Л.И. Исследование влияния модифицирования и микролегирования стали 20ГЛ на ее механические свойства и микроструктуру, Решетневские чтения Сборник 19 конференщии. Красноярск: СибГАУ, 2015, 1, 19, 476-479. 
[Romanova A.A., Pavlov A.V., Nemtsev I.V. Kveglis L.I. Investigation of the effect of modifying and micro-doping steel 20GL on its mechanical properties and microstructure, Reshetnevskie readings, Collection 19 of the Conference, Krasnoyarsk: SibGAU, 2015, 1, 19, 476-479 (in Russian)]

[12] Алабышев А.П. Расчет скорости распространения ультразвука в зависимости от температуры материал, Новые материалы и технологии в машиностроении - 2014: 20-я Международная научно-техническая конференция. Архангельск: ФУ им. М.В. Ломоносова, 2014, 20, 3-5. [Alabyshev A.P. Calculation of the speed of propagation of ultrasound depending on the temperature of the material, New materials and technologies in mechanical engineering - 2014: 20th International Scientific and Technical Conference. Arkhangelsk: FU them. M.V Lomonosov, 2014, 20, 3-5 (in Russian)]

[13] Столяров В.В. Электростимулирование пластической деформаџии объемных наноструктурных титановых сплавов с эффектом памяти формы. Отчет о НИР, ИМАШ РАН. Москва, 2007. [Stolyarov V.V. Electrostimulation of plastic deformation of bulk nanostructured titanium alloys with shape memory effect, Report on research, IMASH RAS, Moscow, 2007 (in Russian)]

[14] Квеглис Л.И., Жигалов В.С., Казанцева В.В., Кузовников А.В., Мусихин В.А., Софронов П.В. Особенности структуры межзеренных границ в сплавах инварного состава на основе Fe-Mn и Fe-Ni. Нанотехника, 2007, 4, 73-78. [Kveglis L.I., Zhigalov V.S., Kazantseva V.V., Kuzovnikov A.V., Musikhin V.A., Sofronov P.V. Features of the structure of grain boundaries in invarbased alloys based on Fe-Mn and Fe-Ni, Nanotechnology, 2007, 4, 73-78 (in Russian)] 